\section{COMBATING CALCULUS}

Oral-B Pro-Expert toothpaste is formulated with polyphosphate which helps prevent the initial build-up of calculus on teeth. This active ingredient inhibits the crystal growth of the unwanted hydroxyapatite in residual plaque, inhibiting mineralisation and helping to prevent calculus formation.

Laboratory and clinical work have confirmed this effect, especially when Oral-B Pro-Expert toothpaste has been used over time. Furthermore its substantial efficacy against calculus has been confirmed.

Of additional benefit to users of Oral-B Pro-Expert toothpaste is the unique

\section{TAILORED X-RAYS}

The Phot-X II is a state of the art, DC $\mathrm{X}$-ray unit enabling the practitioner to tailor every X-ray to the individual needs of the patient.

Delivering the ultimate in multi functionality the Phot-X II has straightforward to use, intuitive controls and a lightweight, easily positioned X-ray head. The scissor arm provides flexibility and total stability ensuring perfectly consistent positioning every time. Additionally the Phot$\mathrm{X}$ II is compatible with most types of digital imaging system and two different film types. Sixteen film speeds are available, delivering sharp, accurate images of superb quality. combination of polyphosphate and stabilised stannous fluoride. In a remarkable synergy, this latter ingredient delivers long-lasting anti-bacterial benefits and as such is recognised as a broad-spectrum antimicrobial agent. Its ability to inhibit bacterial growth, reduce bacterial adhesion and to affect microbial metabolic processes adds the double advantage of anti-plaque to the anti-calculus defences against gum problems.

\section{Reader response number 58}

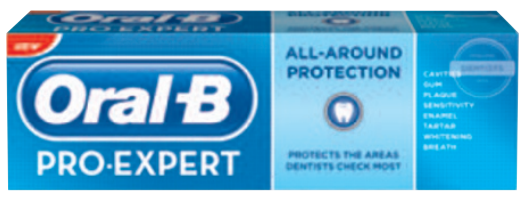

\section{COMPLETE COMPLIANCE} SOLUTION

ResusPod is the complete compliance solution for the management of medical emergencies by the dental team. Medical emergencies are rare but potentially devastating and so ResusPod Limited has recognised the need to support the dental team by providing a comprehensive solution for commonly encountered medical emergencies. The Resuscitation Council (UK) devised the regulations that will now be enforced by the CQC. ResusPod, developed by dentists for dentists, provides a complete solution comprising training, equipment and drugs to ensure that medical emergencies are dealt with effectively and efficiently, simultaneously ensuring compliance obligations are kept up to date.

ResusPod training ensures that all aspects of medical emergency training are dealt with, including the use of appropriate drugs, AEDs and other emergency equipment in addition to basic life support. The emphasis is on scenario-based learning with a team approach. The ResusPod's unique offering covers all regulatory requirements including the CQC's request for accessible, tamper evident containers for medicines and the use of special infection control materials. Sophisticated inventory management tools provide additional peace of mind for every practice registered with ResusPod. In addition, bespoke treatment algorithms (flow charts) support emergency situations by prompting the dental team to take appropriate actions. Reader response number 61

of a Beautifil II restoration. Saliva forms a material film on the surface of Beautifil II that is reported to minimise plaque adhesion and inhibit bacterial colonisation as an ongoing process.

The chameleon effect makes Beautifil II the material of choice for all restorations as the material takes on the colour of the surrounding tooth tissue. With a highly translucent finish this radiopaque versatile, highly dependable material exhibits excellent wear resistance combined with a high filler load to ensure longevity of the final restoration.

Reader response number 60

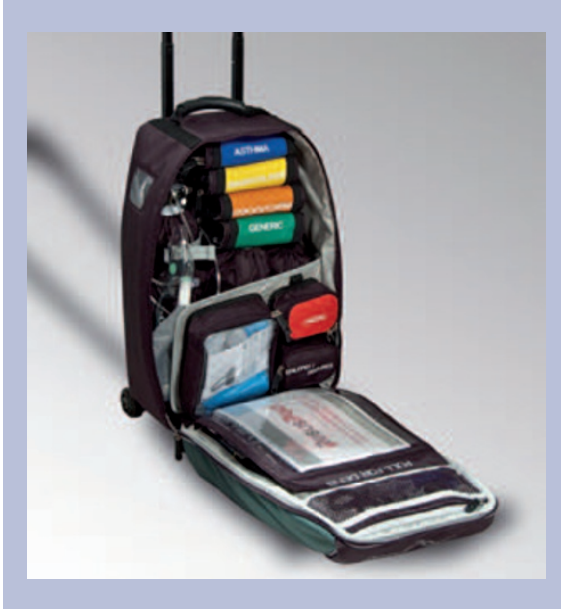

